\title{
EFFECT OF CORONA POWER ON THE CMY REPRODUCTION QUALITY WITH ELECTROINK PRINTED ON FINE ART PAPER
}

\author{
MARKO MORIĆ, IGOR MAJNARIĆ* and MARIO BARIŠIĆ \\ University North, Department of Multimedia, Design and Application, \\ 31b, Jurja Križanića Str., 42000 Varaždin, Croatia \\ "University of Zagreb, Faculty of Graphic Arts, 2, Getaldićeva Str., 10000 Zagreb, Croatia \\ \Corresponding author: Marko Morić,mmoric@unin.hr
}

Received May 24, 2019

\begin{abstract}
In order to achieve high-quality colour prints by using an electrophotography (EP) printing machine, it is necessary to prepare the printing substrate. Additional treatment may be needed to achieve better substrate surface tension for optimally attaching the liquid EP ink to the paper. To ensure good adhesion of the ink to the printing substrate, a corona treatment may be used. During the corona treatment, the surface of the paper is modified, and the ability of paper to accept the ink is improved. In this study, we will determine the possibility of realizing CMY prints and of achieving an increased range of tone value in EP digital printing. Based on the results, the new possible values of reproduced CMY tones will be determined, so that higher print quality can be achieved. The results show that the best colour reproduction is achieved without the use of corona power $(0 \mathrm{~W})$. The additional activation of $450 \mathrm{~W}$ corona will result in the greatest colour variation of $\Delta \mathrm{E}_{\mathrm{C}}=0.60, \Delta \mathrm{E}_{\mathrm{M}}=0.60$ and $\Delta \mathrm{E}_{\mathrm{Y}}=0.48$.
\end{abstract}

Keywords: fine art paper, in-line corona treatment, ElectroInk, digital colour offset

\section{INTRODUCTION}

The treatment process used to modify the top layers of print media is called corona discharge. Even though this procedure was first applied in 1960 for polyethylene film processing, due to the application of dielectric printing substrates and the implementation of single-corona treatment, it would be better to name this procedure as 'dielectric barrier discharge with alternating current at standard atmospheric pressure'. Because it is easy to conduct a corona treatment, it is the most commonly used industrial surface treatment for polyolefin materials. The corona treatment is optimal for successfully treating materials, such as elastomers, synthetic thermoplastic materials and paper. ${ }^{2}$ Corona discharge creates strong surface compatibility with hydrophilic materials and increases adhesion with thin layers, such as coatings, varnishes, adhesives and membranes. A material may return to its initial state after the effect of corona discharge has ceased. ${ }^{3}$ The type and amount of functional groups on a material's surface determines the different contact angles and degree of acceptance of fluids. Additionally, this procedure can be also applied in cleaning aluminum surfaces, as well as in the modification of paper and cardboard surfaces. ${ }^{4}$

\section{THEORETICAL BACKGROUND}

One of the frequently used activating coronas for machines that print from roles is made by a Danish company Vetaphone. Such a unit consists of paper roll rollers, which, with the help of a backing cylindrical electrode and a contact output roller, provide a satisfactory voltage. In the central part, there is a high voltage distribution connection that activates four ceramic electrodes located in a specially adapted housing. This placement ensures the correct distance of the electrodes from the print media. Because the charging process releases harmful ozone, an ozone depletion device is placed above the crown ${ }^{5}$ (Fig. 1).

Achieving high frequency during the corona treatment with four electrodes and four ground electrodes (Vetaphone Corona-Plus device) is possible when the corona is powered with precisely defined voltage. Therefore, the corona unit also contains an appropriate transformer for high frequency regulation. In order to create a 
difference in electric potential and a partial voltage discharge, there should be enough air in the region around the electrodes, and the pressure of air should be around one atmosphere. Only such atmospheric conditions can initiate the ionization process. An important characteristic of the corona discharge is the construction of the corona and the position of thin wire electrodes. As

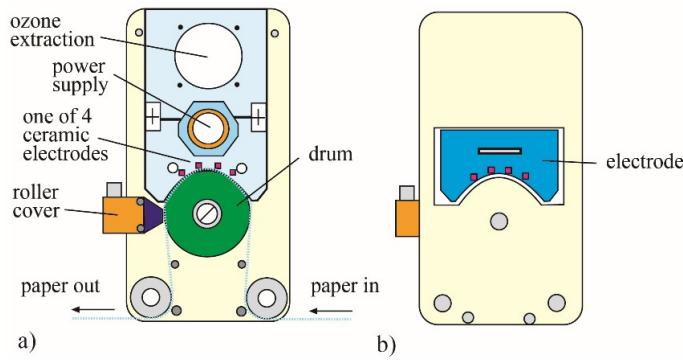

Figure 1: Vetaphone corona used in an HP Indigo machine: a) back; b) front

to additionally consolidate its surface. ${ }^{6}$

In order to achieve the appropriate surface energy on the printed substrate, the substrate must be bombarded with electrons within the space of electrodes. During this treatment, the kinetic energy of the released electrons must be extremely large and it is released by generating an electromagnetic field of about $10 \mathrm{eV}$. The electrons released this way hit the print substrate, and initiate modification of the surface layer and the reduction of the polymer chain. This entails that there is a precondition that must be met: that the electron energy must be greater than the molecular bond energy of the printing medium. The speed of the electromagnetic field change between the electrodes will cause the vibration of the atoms, which will result in ionization and dissociation of the surrounding atmosphere. However, the kinetic energy of particles, plasma atoms and ions generated during the discharge of the corona is much lower than the kinetic energy of the electrons released $\left(10^{-2}\right.$ to $10^{-4}$ times smaller). ${ }^{7}$

The kinetic energy of the electrons in plasma is usually greater than the energy of the basic bonds in the polymer chains of the printing substrate. Thus, the collision of released electrons with polymer chain will lead to the interruption of the chain and to changes in the properties of the upper layer of polymers. ${ }^{8}$ Breaking the surface bonds will result in the formation of radicals that initiate further chemical reactions, such as oxidation and degradation. The difference their power supply can be unipolar or bipolar, the dominance of the ions depends on the coronal polarity. To achieve greater efficiency, the ground electrode is coated with a number of different layers. Thus, the surface of the electrode includes: a polyester layer, a ceramic layer, a silicone rubber layer and epoxy coating. Polyester resin and glass fibers are added in order

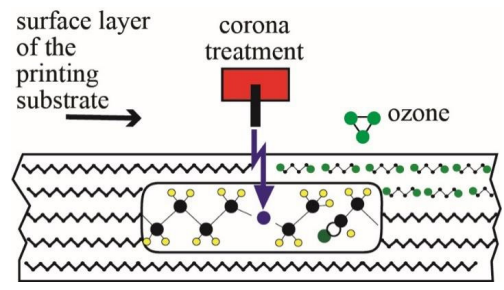

Figure 2: Schematic representation of changes in the top layer of the treated material

between the treated and untreated polymeric substrate is shown in Figure 2. ${ }^{9}$

During the corona discharge, ozone, oxygen atoms and oxygen-free radicals occur. Sometimes, even low temperature plasma can occur. ${ }^{10}$ The outcome of the corona treatment is based on the process of roughening due to the chemical modification of the surface. Micro-roughening causes ablation and surface cleaning by removing organic pollutants due to the relatively low concentration of ionized plasma. The corona treatment plays an important role when it comes to the removal of amorphous and low molecular substances (weak organic and inorganic compounds), thus improving the coating and paint binding properties. The ablation process is important when removing weakly bonded elements from the surface and thus increasing the value of roughness. Thus, the released electrons are lost, so that the $\mathrm{O}_{2}$ and $\mathrm{H}_{2} \mathrm{O}$ particles turn into negative $\mathrm{O}^{-}$and $\mathrm{OH}^{-}$ions. Such ions later interact with positive ions and create new neutral types. ${ }^{11}$

The ozone that emerges during the discharge causes damage (cracks or cuts) on the surface of the intended printing substrate. Ozone formation depends on the polarity of the corona discharge, the relative humidity of the air and the content of the gases in the atmosphere. ${ }^{12}$

The achieved micro-roughness of the upper polymer layer may range from 0.5 to $10 \mathrm{~nm} .{ }^{13}$ As the electrons hit and break down the chemical bonds (C-C and $\mathrm{C}-\mathrm{H}$ ), free radicals are formed in the interrupted polymer chain. Such radicals can 
form new unstable formations, or bomb or link to each other. The main groups that appear are: ketones, aldehydes, hydroxyls and carboxyls. Ultimately, the result is the hydrophilisation of the surface and improved adhesion and acceptance of other layers in a laminated substrate.

Digital colour offset is a specially developed electrophotography technique that utilizes ElectroInk liquid. To generate a single imprint, the machine should contain a three cylinder satellite unit (PIP cylinder, intermediate cylinder and impression cylinder), which serves for indirect transfer of ElectroInk to the print media.

ElectroInk is a special liquid electrophotography ink, which, depending on the colour tone, contains the following components: volatile ISOPAR oil (90\% to 95\%), pigment particles (5\% to $8 \%$ ) and charge regulator Image agent (1\% to $3 \%){ }^{14}$ The liquid ink is prepared immediately prior to printing, using densitometers (the proportion of dry matter content in the liquid) whose value is $1.70 \mathrm{~g} / \mathrm{cm}^{3} .^{15}$ The selective behavior of ElectroInk depends on the established electrostatic field between the developer roller and the PIP cylinder or the PIP cylinder and the intermediate transfer drum. ${ }^{16}$

The liquid ElectroInk is transferred to the heated surface of the intermediate transfer drum, which has a soft offset rubber on its surface. ${ }^{17}$ During that process, ElectroInk changes its aggregate state and turns into a sticky paste. ${ }^{18}$ In order to achieve a complete transfer, the offset rubber cover must meet two basic properties: it must be conducive, and it must be heated to a temperature above $100{ }^{\circ} \mathrm{C}$.

In order to ensure the ability to print in photo quality, BID units should be added containing CMY colours. ${ }^{19}$ A parameter that significantly influences the quality is the adequate print of raster elements that can be directly controlled by activating laser power, ${ }^{20}$ as well as by adjusting the colour transfer changing the voltage on the BID units and by selecting the appropriate printing substrate. $^{21}$

\section{EXPERIMENTAL}

For experimental printing, an HP Indigo WS 6800 was used with an integrated in-line primer injector unit and a corona unit. The RIP (Raster Image Processor) used for separation of colour extracts was an ESCO HPE ProLiant ML350, while using a standard calibration curve LUT 0.5 and 180 lpi raster lines. For testing, the standard print form FOGRA Image Quality of the SRA3 dimension was used, out of which we applied cyan (C), magenta (M) and yellow (Y) separation. ${ }^{22}$

The paper substrate used for the experiment was 90 $\mathrm{g} / \mathrm{m}^{2}$ Condat digital gloss RL (gloss coated fine art paper). The composition of such paper is the following: $50 \%$ virgin cellulose, $40 \%$ calcium carbonate, $4.5 \%$ water and $5.5 \%$ latex and sizing agents. ${ }^{23}$ Other properties of the used fine art paper are shown in Table 1.

For this experiment, a Vetaphone model VE1A-A (C4) 410 corona unit was used. The corona power parameter can be varied, within a range from 0 to 1200 watts. We used three characteristic corona power levels: no power $(0 \mathrm{~W})$, low $(450 \mathrm{~W})$ and high power $(950 \mathrm{~W})$. The technical characteristics of the corona are shown in Table 2.

Because the measurements were conducted under industrial conditions, the surface tension measurement method was applied, in accordance with the norm ASTM D-2578, with the use of indicator fluids containing 2-ethoxyethanol and formamide. In this way, the pause between the examination and testing phases was shortened. Table 3 shows the conditions used during the measurement of surface tension of the treated materials, while using corona power levels of 0 $\mathrm{W}, 450 \mathrm{~W}$ and $950 \mathrm{~W}$. The surface tension of original fine art paper (without corona treatment) was 36 $\mathrm{mN} / \mathrm{m}$, while after corona treatment (corona power of $450 \mathrm{~W}$ and $950 \mathrm{~W}$ ) it achieved $34 \mathrm{mN} / \mathrm{m}$.

In addition to testing CMY reproduction on paper, the corona impact test was also performed on the colour reproduction. To determine the CMY reproducibility, a $4^{\text {th }}$ generation $\mathrm{X}$-rite $\mathrm{eXact}^{24}$ colorimeter and a spectrophotometer were used, while a Personal IAS was used for visual analysis of magnified CMY raster elements on the imprint. ${ }^{25}$ The obtained CIE L*a*b* and CIE LAB $\Delta$ E results were compared to each other using Origin 8.0 software. During experimental printing, different corona power levels were used $(0 \mathrm{~W}, 450 \mathrm{~W}$ and $950 \mathrm{~W})$. As a result, nine different samples were produced during experimental printing, as shown in the schematic representation of the experimental procedure (Fig. 4).

\section{RESULTS AND DISCUSSION}

The quality of electrophotographic prints is strongly influenced by the printing substrate used. The characteristics of the printing substrate are best seen on the FTIR spectrum, which shows absorption in the wavelength range from 600 to $1.900 \mathrm{~cm}^{-1}$. For recording the spectra, an Iraffinity-21 spectrometer was used in the ATR mode, with an incoming angle of $45^{\circ}$ and with the refractive index of the $\mathrm{ZnSe}$ prism of 2.4. For the experimental paper sample, five measurements with a resolution of $4 \mathrm{~cm}^{-1}$ were made in the range from 500 to $4000 \mathrm{~cm}^{-1}$ (Fig. 5). 
The sample does not show the characteristic peaks of cellulose, because of the high content of limestone and other fillers. $\mathrm{CaCO}_{3}$ is predominant with characteristic peaks at 1796, 1373, 1093, 870,712 and $698 \mathrm{~cm}^{-1}$. In the samples numbered
2 and 3, there were two peaks at 2920 and 2850 $\mathrm{cm}^{-1}$, due to calcium stearate $\left(\mathrm{C}_{36} \mathrm{H}_{7} \mathrm{CaO}_{4}\right)$, which is used in combination with starch and urea formaldehyde as a filler in paper and cardboard production.

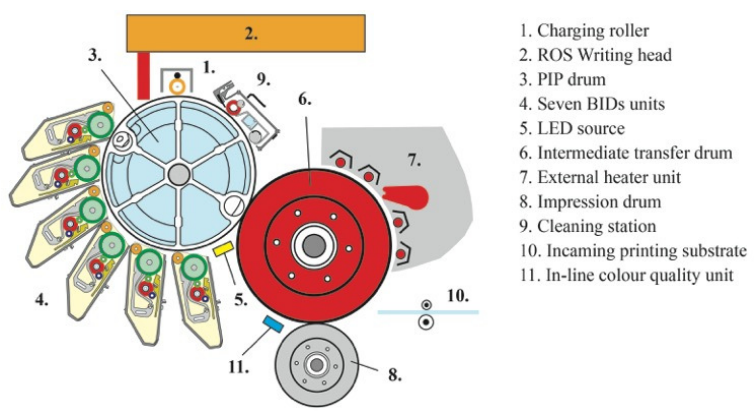

Figure 3: Schematic representation of printing process on digital colour offset (HP Indigo WS 6800 machine)

Table 1

Characteristics of used gloss coated fine art paper (Condat digital gloss RL)

\begin{tabular}{lcccc}
\hline Parameter & $\begin{array}{c}\text { Unit of } \\
\text { measurement }\end{array}$ & Value & Tolerance & Standard \\
\hline Grammage & $\mathrm{g} / \mathrm{m}^{2}$ & 90 & $\pm 4 \%$ & ISO 536 / TAPPI T410 \\
Thickness & $\mu \mathrm{m}$ & 70 & $\pm 4 \%$ & ISO 534 / TAPPI T411 \\
Opacity & $\%$ & 89 & -2.0 & ISO 2471 / TAPPI T519 \\
Whiteness D65/10 & $\%$ & 118.7 & \pm 3 & ISO 11475 \\
Gloss of paper & $\%$ & 70 & \pm 5 & ISO 8254 / TAPPI T480 \\
Smoothness by Bekk & $\mathrm{s}$ & 1400 & $\pm 30 \%$ & ISO 5627 / TAPPI T475 \\
Relative humidity & $\%$ & 40 & \pm 7 & TAPPI T502 \\
\hline
\end{tabular}

Table 2

Technical characteristics of VE1A-A(C4)410 Vetaphone corona unit

\begin{tabular}{lc}
\hline Parameter & Characteristic \\
\hline & $30 \mathrm{~m} / \mathrm{min}$ for four-color prints \\
Speed of impalement & $40 \mathrm{~m} / \mathrm{min}$ in higher productivity mode \\
& $60 \mathrm{~m} / \mathrm{min}$ for printing one or two colors \\
Length of treatment & $350 \mathrm{~mm}$ \\
Width of roller for treatment & $410 \mathrm{~mm}$ \\
Housing width & $455 \mathrm{~mm}$ \\
Min. space of manipulation & $890 \mathrm{~mm}$ \\
Possibility of treatment & One side \\
Number of electrodes & 4 (ceramic) \\
Roller cover & Stainless steel \\
Roll diameter & $\varnothing 100 \mathrm{~mm}$ \\
Construction type & Mounted on the console or on the base \\
\hline
\end{tabular}


Table 3

Climatic conditions in the production plant during the measurement

\begin{tabular}{lcc}
\hline Samples & $\begin{array}{c}\text { Temperature, } \\
{ }^{\circ} \mathrm{C}\end{array}$ & $\begin{array}{c}\text { Relative humidity, } \\
\%\end{array}$ \\
\hline Paper-corona 0 W & 24.2 & 49 \\
Paper-corona 450 W & 25.1 & 53 \\
Paper-corona 950 W & 25.3 & 53 \\
Min. & 24.2 & 49 \\
Max. & 25.3 & 53 \\
Average & 24.75 & 51 \\
\hline
\end{tabular}

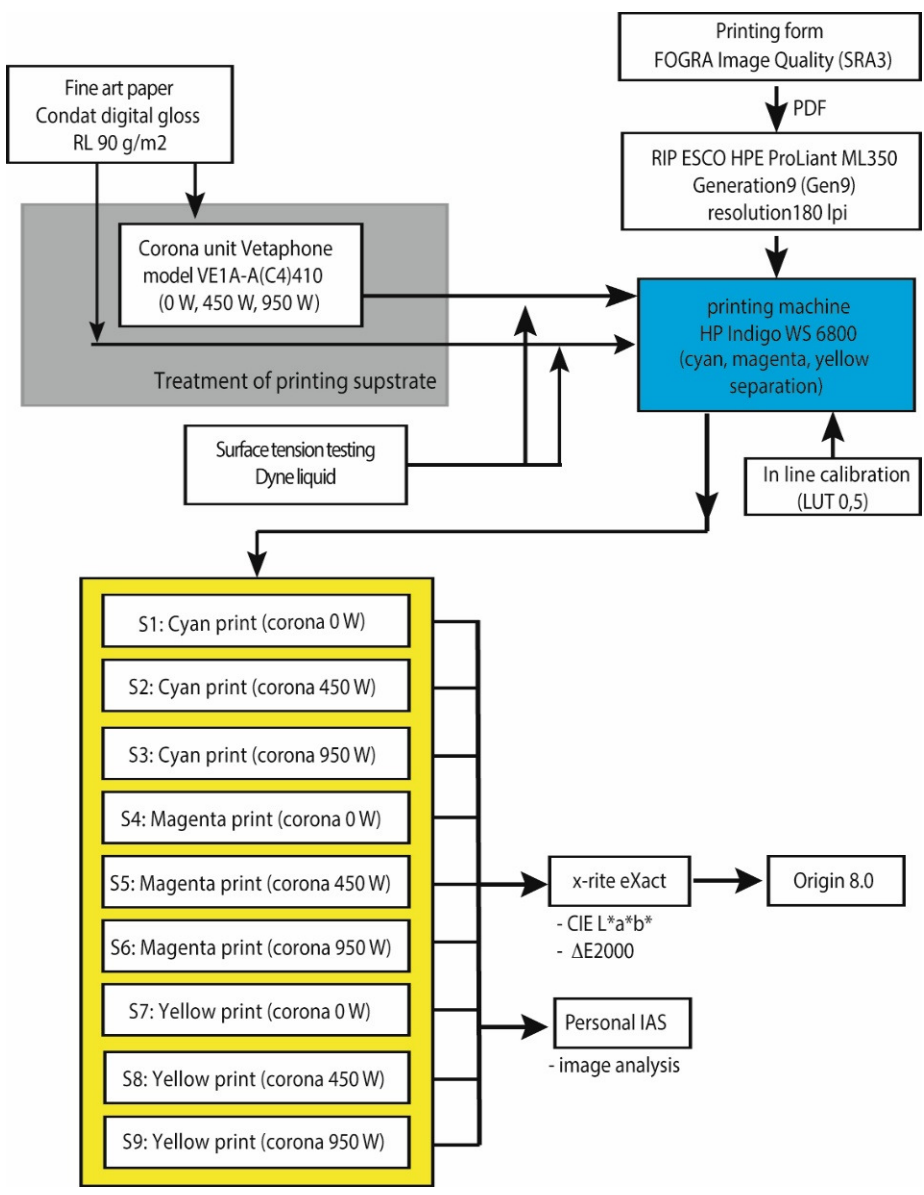

Figure 4: Schematic representation of the chronological experiment 


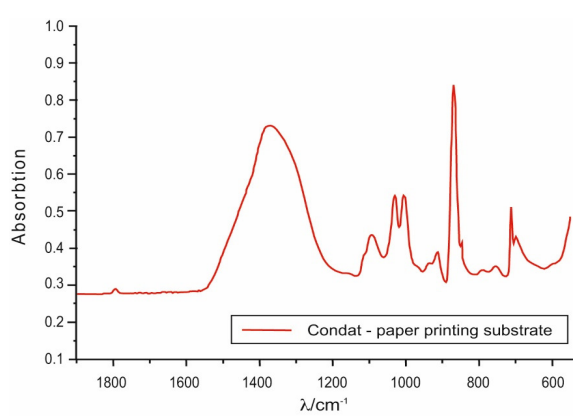

Figure 5: FTIR spectrum of Condat paper for artistic printing obtained on a Shimadzu IRAffinity-1 FTIR spectrometer

Kaolin is the most commonly used filler in the paper industry, primarily due to its key role in increasing opacity and whiteness, while it simultaneously improves colour absorption and stands out sharply at the wavelengths of $1029 \mathrm{~cm}$ ${ }^{1}, 1005 \mathrm{~cm}^{-1}, 790 \mathrm{~cm}^{-1}$ and $756 \mathrm{~cm}^{-1}$ in all the samples.

In order to achieve better adhesion of the ElectroInk, the surface tension of the printing substrate should be kept as low as possible. One of the most successful methods is treating the surface of the paper with plasma generated during corona discharge. By applying the colorimetric measurement method, it is possible to predict the success of binding the printing ink, whereby more intense application will result in higher chromatic saturation (better print). The colour space used for comparison is CIE L*a*b*. The metrics used for comparing two colour coordinates is CIE Lab $\Delta \mathrm{E}$, which provides a visual representation of the colour deviation. Thus, the colour deviation of $\Delta \mathrm{E}$ $<1$ represents visually invisible colour changes, $\Delta \mathrm{E}$ between 1-3 means colour areas within the standard, $\Delta \mathrm{E}$ between 3-5 means the allowable colour deviation range, and $\Delta \mathrm{E}>5$ is a nonpermissible range. The most accurate formula for calculating the colour deviation is CIE Lab $\Delta \mathrm{E} 2000{ }^{26}$

Figures 6, 7 and 8 show the colour values and colour deviations of CMY ElectroInk prints on fine art paper that was previously treated with corona power of $0 \mathrm{~W}, 450 \mathrm{~W}$ and $950 \mathrm{~W}$. Also, Personal IAS images of prints show characteristic surface coverage $(10 \%, 20 \%, 40 \%, 70 \%$ and $100 \%$ tone value (TV)).

There was no visible colour difference on pure fine art paper (Condat $90 \mathrm{~g} / \mathrm{m}^{2}$ ) when corona voltages of $450 \mathrm{~W}$ and $950 \mathrm{~W}$ were used. The curves of the reproduction remain completely linear and the examined areas of $10 \%, 20 \%, 40 \%$, $70 \%$ and $100 \% \mathrm{TV}$ do not exceed the $\Delta \mathrm{E}=1$ colour difference. The resulting colour changes $\left(\Delta \mathrm{E}_{0 \mathrm{~W} \_450 \mathrm{~W}}=0.60 ; \Delta \mathrm{E}_{0 \mathrm{~W} \_950 \mathrm{~W}}=0.37\right)$ are not visible to the human eye. The colorimetric measurement method helped reveal the tendency of surfaces with less coverage (bright tones) to achieve greater colour changes, while increasing the screen tone value reduces this change. The changes were more evident when corona power of $450 \mathrm{~W}$ was used.

Microscopic images demonstrate that corona treatment, with varying corona power levels, does not significantly affect the reproduction of solid tones $(100 \% \mathrm{TV})$. The best print of lower tone values was achieved without the effect of the corona. The raster elements are most clearly visible in the most appropriate cylindrical shape on such prints. This precision is particularly visible in areas of $40 \% \mathrm{TV}$, where one can still spot whiteness between the reproduced raster elements. By activating the corona power, the cyan image becomes blurrier, and in the area of $40 \% \mathrm{TV}$, the raster elements connect into one line. Such an anomaly is caused by changes in the structure of the paper surface, which thus achieves an elliptic raster element because of its roughness (Fig. 6). 

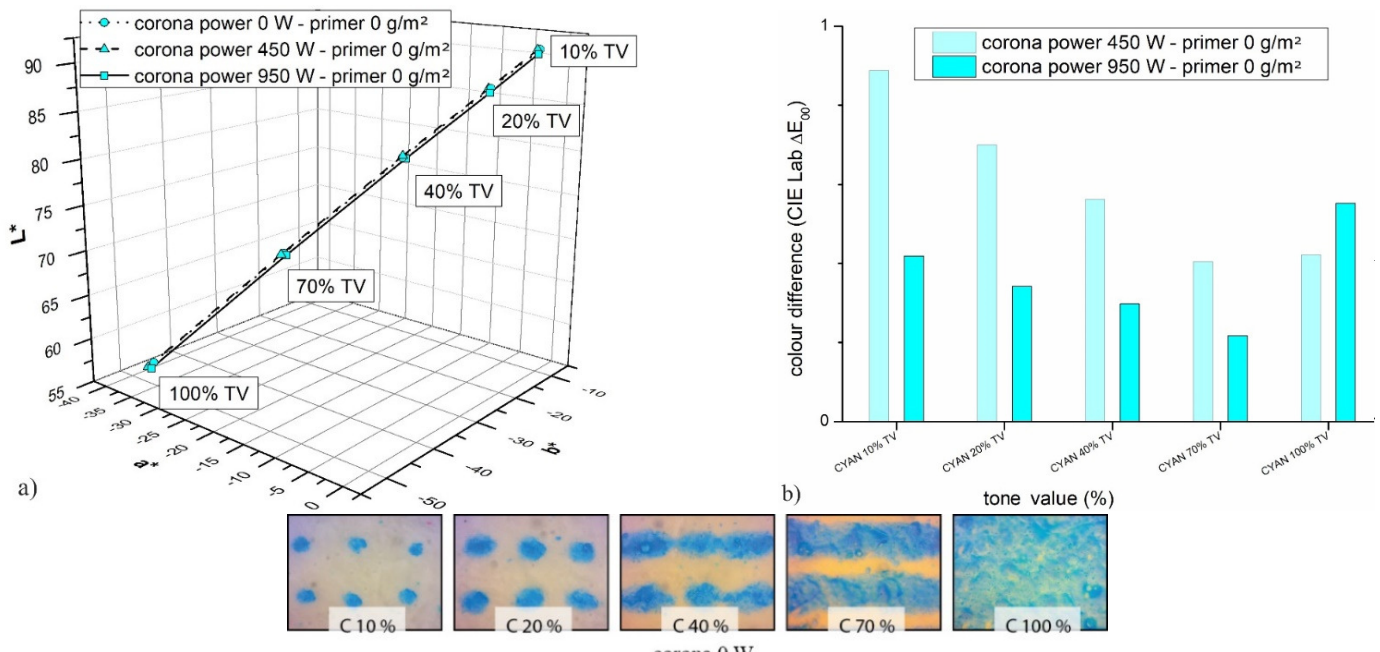

b)
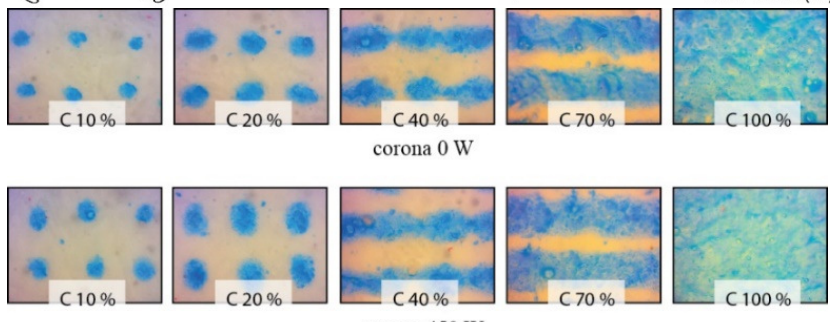

corona $450 \mathrm{~W}$
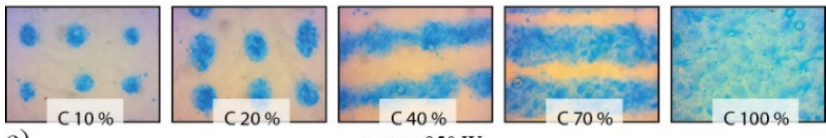

c) corona $950 \mathrm{~W}$

Figure 6: Colour changes of cyan prints, as a result of applying $0 \mathrm{~W}, 450 \mathrm{~W}$ and $950 \mathrm{~W}$ corona voltage variations: a) CIELab colour display; b) $\Delta E$ colour deviations; c) microscopic images of the samples
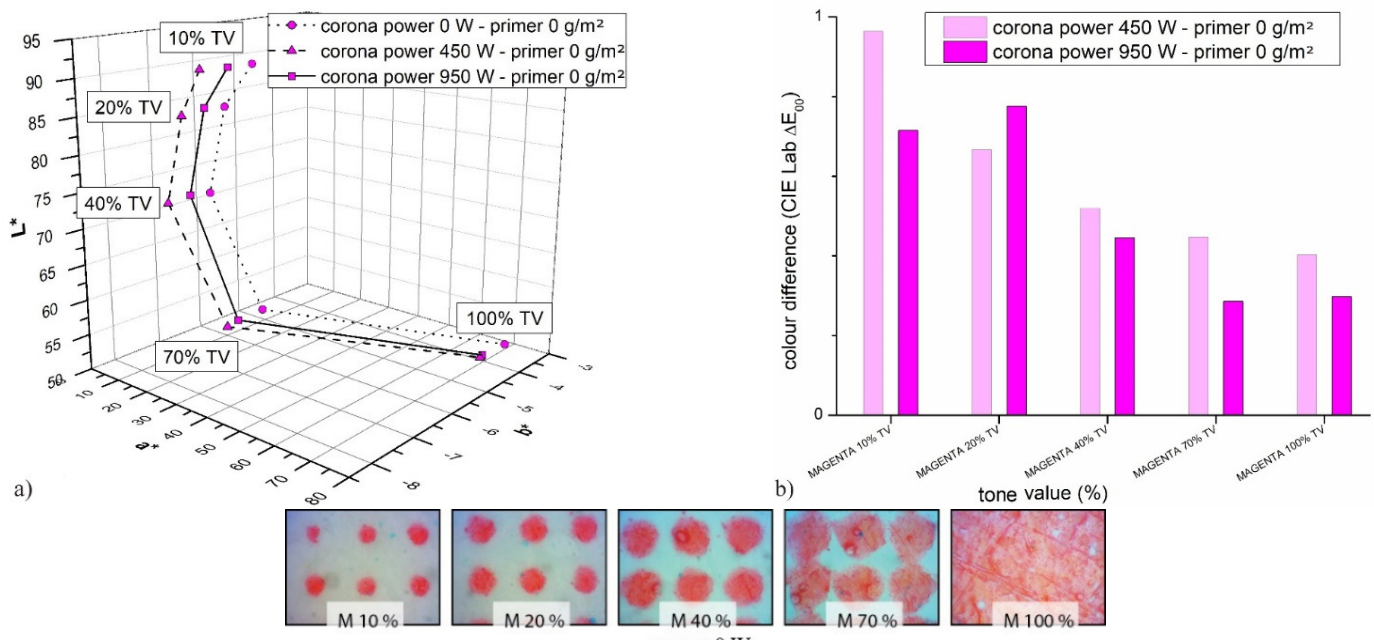

corona $0 \mathrm{~W}$
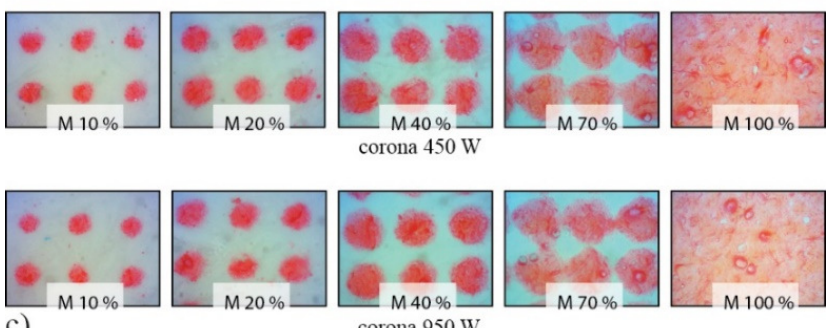

Figure 7: Colour changes of magenta prints, as a result of applying $0 \mathrm{~W}, 450 \mathrm{~W}$ and $950 \mathrm{~W}$ corona voltage variations: a) CIELab colour display; b) $\Delta$ E colour deviations; c) microscopic images of the samples 
In experimental magenta prints, there is no noticeable colour change on the paper without primer, treated using $450 \mathrm{~W}$ and $950 \mathrm{~W}$ corona power. The TV of the examined areas does not exceed the visual colour difference, and it is measured as $\Delta \mathrm{E}_{0 \mathrm{~W} \_450 \mathrm{~W}}=0.60 ; \Delta \mathrm{E}_{0 \mathrm{~W} \_950 \mathrm{~W}}=0.50$. As in the cyan prints, a colour difference was observed in bright tonal values, and it is more evident when using corona power of $450 \mathrm{~W}$ $\left(\Delta \mathrm{E}_{\mathrm{C} 10 \% \_\mathrm{M} 10 \%}=0.07\right)$.

Compared to cyan, magenta ElectroInk is better applied on fine art paper, which is visible in medium tonal areas (screen elements in the area of $40 \% \mathrm{TV}$ remained correct and separated). Using corona power also resulted in the best colour results, with raster points remaining rounded, with the expressed coefficient value (higher application of the ink). As regards the solid tones, it is evident that the increase in corona power reduces the values of solid tones and such a surface will become less contrasting (Fig. 7).

In yellow colour separation, there is no significant colour change when $450 \mathrm{~W}$ and $950 \mathrm{~W}$ corona power was used, without primer. The curves of the reproduction remain linear and the examined areas achieve a colour change of $\Delta \mathrm{E}<$ 0.7 , so that, even in the yellow prints, the resulting colour deviation is not visible to the human eye. Their values are $\Delta \mathrm{E}_{0 \mathrm{w} \_450 \mathrm{w}}=0.48$; $\Delta \mathrm{E}_{0 \mathrm{~W} \_950 \mathrm{~W}}=0.49$.
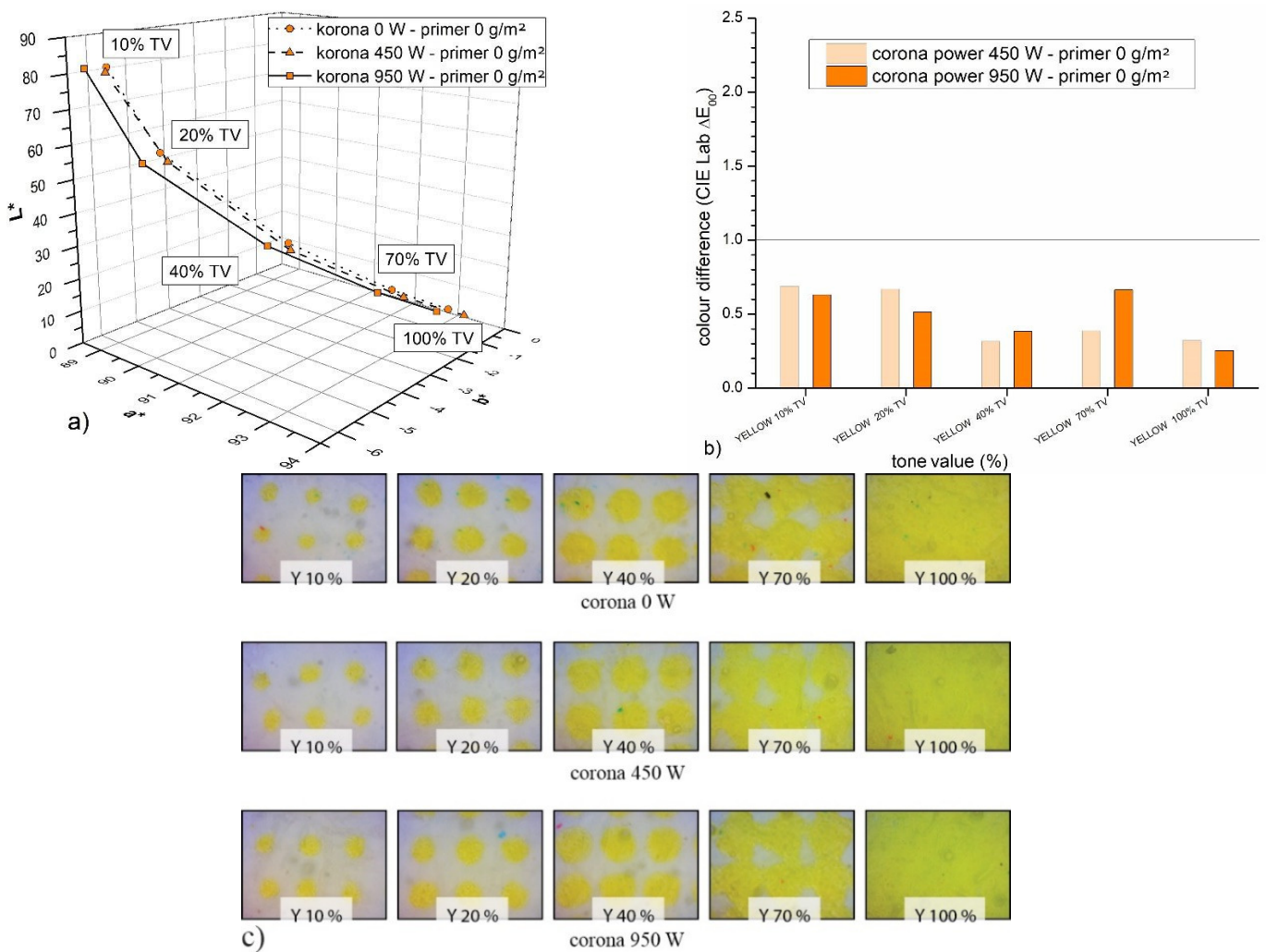

Figure 8: Colour changes of yellow prints, as a result of applying $0 \mathrm{~W}, 450 \mathrm{~W}$ and $950 \mathrm{~W}$ corona voltage variations: a) CIELab colour display; b) $\Delta \mathrm{E}$ colour deviations; c) microscopic images of the samples

The yellow colour separation acts similarly to the magenta print. The smallest and most accurate printing elements (yellow raster dots) are also reproduced for $0 \mathrm{~W}$ corona power treatement. By activating and increasing the corona power supply, the TV will increase, which is particularly noticeable in the higher tone areas (70\%). This results in elements connecting to each other and forming larger surfaces (shapes). It is also evident that the solid tones oscillate, and that the increase in corona power decreases these values (Fig. 8). 


\section{CONCLUSION}

The difference between the two colour changes in cyan prints, due to the variation of corona power $(450 \mathrm{~W}$ and $950 \mathrm{~W})$, is $\Delta \mathrm{E}_{(450 \mathrm{~W}}$ and $950 \mathrm{w})=0.23$. The difference in magenta prints, due to the variation of the experimental voltage of corona power, is $\Delta \mathrm{E}_{(450 \mathrm{w} \text { and } 950 \mathrm{w})}=0.10$. Given the variation of corona power, the difference between the two colour changes in the yellow prints is $\Delta \mathrm{E}_{(450 \mathrm{w} \text { and } 950 \mathrm{w})}=0.01$. Thus, the best separation of elements is reproduced with magenta and yellow ElectroInk. Also, corona treatment is not advisable for the purpose of printing on fine art paper whose coating is based on $\mathrm{CaCO}_{3}$, because it does not significantly influence the reproducibility of CMY prints based on the digital colour offset technology. Although the changes incurred by the corona treatment are not visible, they are important in the whole process of calibrating the electrophotography printing machine. In combination with other parameters of the electrophotography printing process, these changes still affect the final colour results.

\section{REFERENCES}

J. Izdebska, "Printing on Polymers", Elsevier Inc., 2015, pp. 123-142, http://dx.doi.org/10.1016/B978-0323-37468-2.00008-7

2 M. Tuominen and J. Lahti, in Procs. $11^{\text {th }}$ TAPPI European PLACE Conference, Athens, May 14-16, 2007, pp. 1-15, https://tappi.org/

3 T. L. Andres, L. Bufalino, P. I. C. C. Mendes, M. A. Martins, G. H. D. T. Marin et al., Cellulose, 25, 5017 (2018), http://dx.doi.org/10.1007/s10570-0181948-3

${ }^{4}$ B. Mesic, M. Lestelius and G. Engström, Packag. Technol. Sci., $\quad \mathbf{1 9}, \quad 61 \quad$ (2006), http://dx.doi.org/10.1002/pts.708

S. Ovaska, R. Rinkunas, T. Lozovski, R. Maldzius and J. Sidaravicius, J. Appl. Packag. Res., 8, 68 (2016), http://dx.doi.org/10.14448/japr.08.0016

6 M. Tuominen, J. Lahti, J. Lavonen, T. Penttinen, J. P. Räsänen et al., J. Adhes. Sci. Technol., 24, 471 (2010),

http://dx.doi.org/10.1163/016942409X1256125229222 4

S. S. Ovaska, P. Geydt, R. Rinkunas, T. Lozovski, R. Maldzius et al., Polym. Polym. Compos., 25, 257 (2017), https://doi.org/10.1177/096739111702500402

8 Vetaphone, "Corona treatment" (2018), Available at: http://www.vetaphone.com/technology/coronatreatment/

9 M. Niaounakis, "Biopolymers: Processing and Products", Elsevier Inc., 2015, pp. 303-326, https://doi.org/10.1016/B978-0-323-26698-7.00008-8
10 J. R. Rocca-Smith, Th. Karbowiak, E. Marcuzzo, A. Sensidoni, F. Piasente et al., Polym. Degrad. Stabil., $\quad$ 132, $109 \quad$ (2016), https://doi.org/10.1016/j.polymdegradstab.2016.03.020

11 H. Y. Park, B. J. Kang, D. Lee and J. H. Oh, Thin Solid Films, 546, $162 \quad$ (2013), http://dx.doi.org/10.1016/j.tsf.2013.03.067

12 J. W. Gooch, in "Encyclopedic Dictionary of Polymers", edited by J. W. Gooch, Springer-Verlag, 2011, pp. 822-828, http://dx.doi.org/10.1007/978-14419-6247-8_6497

13 Y. Kusano, J. Adhes., 90, 755 (2014), http://dx.doi.org/10.1080/00218464.2013.804407

14 L. Benzion, P. Ben-Auraham, J. Hall and G. A. Gibson, US Patent US4794651A, 1988, p. 18, https://patents.google.com/patent/US4794651

15 A. Yaacov, US Patent US5047306A, 1991, p. 6, https://patents.google.com/patent/US5047306A/en

$16 \mathrm{P}$. Forgacs and A. Teishev, J. Imaging Sci. Technol., $\quad 56, \quad 1 \quad$ (2013), http://dx.doi.org/10.2352/J.ImagingSci.Technol.2012.5 6.4.040573

17 I. Majnaric, K. Golubovic, S. Bolanca and D. Modric, in DAAAM International Scientific Book, Vienna, December 8-10, 2010, pp. 509-524, http://dx.doi.org/10.2507/daaam.scibook.2010.45

18 I. Majnarić, I. Bolanča, Z. Bolanča and M. Milković, in Procs. International Conference on Digital Production Printing and Industrial Applications DPP2005, Amsterdam, May 9-13, 2005, pp. $96-99$

I. Majnaric, D. Modric, and K. Golubovic, in Procs. International DAAAM Symposium, Vienna, October 22-25, 2008, pp. 781-782

20 I. Majnaric, Z. Bolanca and I. B. Mirkovic, in Procs. International DAAAM Symposium, Vienna, November 25-28, 2009, pp. 607-608

21 I. Majnarić, A. Hladnik, T. Muck and I. B. Mirković, Teh. Vjesn., 22, $145 \quad$ (2015), http://dx.doi.org/10.17559/TV-20140321230455

22 A. Kraushaar, "Process Standard Digita", Fogra Research Institute for Media Technologies, 2018, pp. 7-19

23 Lecta group, "Condat data sheet," 2018, Available at:

https://cmspro.lecta.com/Digital/LectaProductCertifica tes/SafetyDataSheet_Condat.pdf

24 X-rite eXact Spectrophotometer, 2018, Available at: $\quad$ https://www.xrite.com/categories/portablespectrophotometers/exact

${ }^{25}$ QEA, Personal IAS, 2018, Available at: http://www.qea.com/upload/files/products/datasheet_p ersonalias-newaddr.pdf

${ }^{26}$ W. Mokrzycki and M. Tatol, Mach. Graph. Vis., 20, $383 \quad$ (2011), https://pascalfrancis.inist.fr/vibad/index.php?action=getRecordDetai 1\&idt=27834549 